\title{
Is prediction of local recurrence possible in Paget's disease of the breast?
}

\author{
SEVIM TURANLI ${ }^{1}$ \\ ${ }^{1}$ SBU, Ankara Oncology Education and Research Hospital
}

January 26, 2021

\begin{abstract}
Purpose Mammary Paget disease of the breast is rare form of breast cancer. Recent trends in the management have changed from mastectomy to breast conserving surgery, its safety is still controversial. The study aimed to predict the patients prone to local recurrence, and to find out the answer to the question of who should be a candidate for breast conserving surgery. Methods Between January 2007 and December 2017, 69 patients who underwent surgery and pathology report diagnosed as Paget's disease, were analyzed retrospectively. As factors that may affect local recurrence; age, presence of nipple symptoms, mass detection in radiological imaging, type of the surgery, stage, pathological findings of the tumor (histological type, multicentricity, ER, PR, HER2, Ki 67 proliferative index, molecular subtype) were evaluated. Results Among 69 patients, 26 patients had ductal carcinoma in situ and others had invasive ductal carcinoma. Number of the patients underwent breast conserving surgery and mastectomy was 15 and 54 respectively. Patients were followed-up for a median time of 45 months. Recurrence was developed in 12 patients, half of them with local recurrence. Type of the surgery was the unique factor influencing local recurrence $(\mathrm{p}=0.001)$. Subgroup analysis was performed among patients undergoing breast conserving surgery, only Ki 67 proliferative index above $30(\mathrm{p}=0.002)$ was found to affect local recurrence. Conclusion Although the number of patients is small, it should be kept in mind that local recurrence may be high when BCS is performed in patients with the tumor's Ki 67 proliferative index is above 30 .
\end{abstract}

\section{Hosted file}

Manuscript File.pdf available at https://authorea.com/users/391929/articles/505933-isprediction-of-local-recurrence-possible-in-paget-s-disease-of-the-breast 